\title{
3D-printed simulator for nasopharyngeal swab collection for COVID-19
}

\author{
Nicolas Sananès ${ }^{1,2}\left(\right.$ Massimo Lodi $^{1,3} \cdot$ Antoine Koch $^{1} \cdot$ Lise Lecointre $^{1,4} \cdot$ Axel Sananès $^{1} \cdot$ Nicolas Lefebvre $^{5}$. \\ Christian Debry ${ }^{2,6}$
}

Received: 7 October 2020 / Accepted: 21 October 2020 / Published online: 6 November 2020

(c) Springer-Verlag GmbH Germany, part of Springer Nature 2020

\begin{abstract}
Introduction Testing for COVID-19 is a cornerstone of pandemic control. If conducted inappropriately, nasopharyngeal swab collection can be painful and preanalytical sample collection errors may lead to false negative results. Our objective was to develop a realistic and easily available synthetic simulator for nasopharyngeal swab collection.

Materials and methods The nasopharyngeal swab collection simulator was designed through different development steps: segmentation, computer-aided design (CAD), and 3D printing. The model was 3D printed using PolyJet technology, which allows multi-material printing using hard and soft materials.

Results The simulator splits in the parasagittal plane close to the septum to allow better visualization and understanding of nasal cavity landmarks. The model is able to simulate the softness and texture of different structural elements. The simulator allows the user to conduct realistic nasopharyngeal swab collection. A colored pad on the posterior wall of the nasopharynx provides real-time feedback to the user. The simulator also permits incorrect swab insertion, which is of obvious benefit from a training perspective. Comprehensive 3D files for printing and full instructions for manufacturing the simulator is freely available online via an open access link.

Conclusion In the context of the COVID-19 pandemic, we developed a nasopharyngeal swab collection simulator which can be produced by $3 \mathrm{D}$ printing via an open access link, which offers complete operating instructions.
\end{abstract}

Keywords Coronavirus · COVID-19 $\cdot$ Nasopharyngeal swab collection $\cdot$ Simulator $\cdot$ Training

\section{Introduction}

Nicolas Sananès

nicolas.sananes@chru-strasbourg.fr

1 Obstetrics Department, Strasbourg University Hospital, Strasbourg, France

2 INSERM UMR-S 1121 'Biomaterials and Bioengineering', Strasbourg University, Strasbourg, France

3 CNRS UMR7104, INSERM U964, Institute of Genetics and Molecular and Cellular Biology (IGBMC), Illkirch-Graffenstaden, France

4 I-Cube UMR 7357, laboratoire des Sciences de l'ingénieur, de l'informatique et de l'imagerie, Strasbourg University, Strasbourg, France

5 Infectious Diseases Department, Strasbourg University Hospital, Strasbourg, France

6 ENT Department, Strasbourg University Hospital, Strasbourg, France
Testing for COVID-19 is a cornerstone of pandemic control all over the world, since it allows better follow-up of the outbreak, isolation of those who test positive, and contact tracing [1]. The number of SARS-CoV-2 tests performed daily in the United States has dramatically increased to $800,000-1,000,000$ at the beginning of October, and a total of more than 100 million tests have been carried out since the epidemic began [2]. Dedicated clinical test sites have begun to spring up such as drive-through test centers. Finally, there is increased availability of testing, with expanding numbers and types of health care providers who conduct these tests.

For initial diagnostic testing for SARS-CoV-2, the recommendation of the Centers for Disease Control and Prevention (CDC), in their latest interim guidelines, is to collect and test an upper respiratory specimen. Nasopharyngeal swab collection (NP-SC) can be performed 
simply by a healthcare provider: it has few contraindications and can easily be rolled out on a large scale [3]. If conducted inappropriately however, this test can be painful and preanalytical sample collection errors may lead to false negative results: the subsequent fall-off in sensitivity has major epidemiological implications [4].

There is therefore a need for appropriate training of healthcare providers likely to be involved in testing. Relevant teaching content is already available online, such as the video "How to Obtain a Nasopharyngeal Swab" [5]. Nevertheless, there is still a need for practical training to avoid patient discomfort and to ensure conditions that are safe for the healthcare provider, i.e., without infectious risk.

Our objective was to develop a realistic and easily available synthetic simulator for nasopharyngeal swab collection.

\section{Methods}

The NP-SC simulator was designed to high-level standards by BONE 3D (Paris, France). The different development steps were segmentation, computer-aided design (CAD), and $3 \mathrm{D}$ printing. The segmentation step was performed using anonymized CT- and MRI-scans of the nasal and pharyngeal cavities using open-source software (3D Slicer software, version 4.10.2). The segmented elements were then reconstructed using CAD software (3-Matic 14.1, Materialise, France). Finally, the simulator was designed to include main anatomic landmarks: nasal bones (including turbinates), nasopharynx, hard palate, facial skin, and mucosa.

The model was 3D printed using PolyJet technology (J750 ${ }^{\mathrm{TM}} 3 \mathrm{D}$ printer, Stratasys, Israel). Among the various 3D print technologies, this particular technique allows multimaterial printing using hard and soft materials with a Shore A hardness of between 30 and 90 (i.e., from very soft and flexible to less soft with no flexibility). The material selected for each anatomic part was based on its Shore hardness and color. The resultant model is thus able to simulate the softness and texture of different structural elements, notably the smooth mucosal lining over hard bone, and the pliable nasopharynx.

The model was also 3D printed using fused deposition modeling (FDM) technology (MethodX Desktop ${ }^{\mathrm{TM}}$ 3D-printer, Makerbot, New York, USA). This technique does not allow multi-material printing, but is less expensive and more accessible than PolyJet technology. This version of the model was printed in a thermoplastic polymer, acrylonitrile butadiene styrene (ABS), and is entirely stiff. The anatomy has been slightly modified to make swab insertion possible despite the lack of pliability.

\section{Results}

The simulator is illustrated in Fig. 1. The model splits in the parasagittal plane close to the septum in order to allow better visualization and understanding of nasal cavity landmarks. Both parts have magnetic contacts, so they can readily be assembled and separated. Nasopharyngeal swab collection can be performed from either nostril. Slots on the posterior wall of the nasopharynx allow fitting of a replaceable colored pad. By coloring the swab tip, it provides real-time feedback to the user.

A video presenting the simulator is freely available online via this link: https://landing.bone3d.com/np-swabsimulator. It gives an account of the different landmarks in the nasal cavity and demonstrates how to use the simulator. It does not describe the specific set-up and equipment required for nasopharyngeal swab collection or specimen handling, which can be found elsewhere [5].

The simulator allows the user to conduct realistic nasopharyngeal swab collection by inserting the swab through the nostril, parallel to the palate, and gently advancing it horizontally down to the nasopharynx, where the colored pad is located. The swab should then be withdrawn in the same axis. We recommend the use of petroleum jelly or a suitable lubricant to facilitate gliding of the swab. The simulator can be rinsed with water. The simulator also permits incorrect swab insertion (typically towards the higher part of the nasal cavity), which is of obvious benefit from a training perspective.

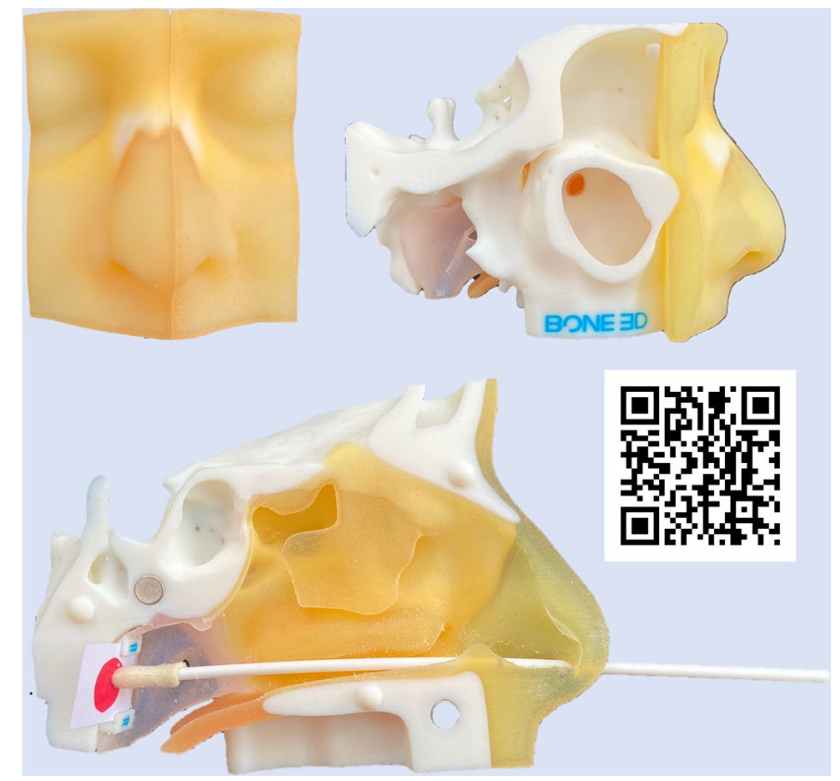

Fig. 1 Pictures of the nasopharyngeal swab collection simulator 


\section{Discussion}

Currently, 3D printing is gaining momentum and is becoming more and more common in different fields such as engineering, architecture, industry, aviation, research, and healthcare. 3D printing typically uses different materials such as plastics, resin, and metal. Since we wanted the NP-SC simulator to be easily and freely shared worldwide, we devised two versions, a highly realistic model and a less realistic model.

The first model is more realistic since it is 3D printed in multi-materials as described above. However, it is more expensive and less readily available. It can only be printed by a PolyJet 3D printer expert working in a university department or research facility, or professionally by a company specialized in $3 \mathrm{D}$ printing.

The second model is simpler, being made of a single type of plastic and printable at a lower resolution. The advantages of this model are that it is cheap and can be made by a nonprofessional 3D printer using FDM technology, in fabrication laboratories ("fab labs"), or even at home.

Although the first model is more realistic, nasopharyngeal swab collection can be performed with both simulators, which display all the main anatomic landmarks. Comprehensive 3D files for printing and full instructions for manufacturing both models are freely available online via this link: https://landing.bone3d.com/np-swab-simulator.

To be noted that this simulator does not address septal deviations or inferior turbinate hypertrophy. Unfortunately, it was not possible to design variations and users should be aware that this simulator does not stimulate septal deviations.

A recent study showed the potential interest of anterior samples (tongue, nasal, or mid-turbinate), which can be collected by patients for the diagnosis of COVID-19 [6]. The simulator we present could also be a way for self-collecting nasopharyngeal sample.

In conclusion, in the context of the COVID-19 pandemic, we developed a nasopharyngeal swab collection simulator which can be produced by 3D printing via an open access link. This link offers complete operating instructions. We have also provided an online video showing how to use the simulator. Our hope is that this training tool for healthcare providers will boost accuracy and improve patient comfort during nasopharyngeal swab collection.

Acknowledgements The authors are grateful to Juliette Prebot, Lucie Arboy-Klein, and the BONE 3D team for enabling them to make the highly technical simulator file freely available online. The authors would also like to thank Marc Augustin and Philippe Bastide of Dianosic for their insights and contributions over the course of the project.

Funding This project was funded by "Protège Ton Soignant", a French social non-profit organization providing material assistance to caregivers during the COVID-19 pandemic.

\section{Compliance with ethical standards}

Conflicts of interest All the authors declare they have no conflicts of interest.

\section{References}

1. Fineberg HV (2020) Ten weeks to crush the curve. N Engl J Med 382(17):e37. https://doi.org/10.1056/NEJMe2007263

2. COVID Tracking Project (2020). https://covidtracking.com/api. Accessed 05 Oct 2020

3. Centers for Disease Control and Prevention (2020) Interim guidelines for collecting, handling, and testing clinical specimens for COVID-19. https://www.cdc.gov/coronavirus/2019-ncov/lab/ guidelines-clinical-specimens.html. Accessed 08 Oct 2020

4. Woloshin S, Patel N, Kesselheim AS (2020) False negative tests for SARS-CoV-2 infection-challenges and implications. N Engl J Med. https://doi.org/10.1056/NEJMp2015897

5. Marty FM, Chen K, Verrill KA (2020) How to obtain a nasopharyngeal swab specimen. N Engl J Med 382(22):e76. https:// doi.org/10.1056/NEJMvem2010260

6. Tu YP, Jennings R, Hart B, Cangelosi GA, Wood RC, Wehber K, Verma P, Vojta D, Berke EM (2020) Swabs collected by patients or health care workers for SARS-CoV-2 testing. N Engl J Med 383(5):494-496. https://doi.org/10.1056/NEJMc2016321

Publisher's Note Springer Nature remains neutral with regard to jurisdictional claims in published maps and institutional affiliations. 